\title{
Estrategias cognitivas y metacognitivas para el desarrollo de capacidades en el área de matemática en los niños y niñas del quinto grado de primaria de la Institución Educativa No 16139 Sallique - Jaén -Perú.
}

Cognitive and metacognitive strategies for the development of capacities in the mathematica area in the children of the fifth grade of Primary School $N^{\circ} 16139$ Sallique - Jaen - Perú.

\author{
'Yovany Sembrera C., , 'Neyla Rubio V. a , 'Julio Alvarado M., ${ }^{2}$ Carlos Núñez R.
}

\section{RESUMEN}

Esta investigación tiene como propósito aplicar estrategias cognitivas y metacognitivas para desarrollar capacidades significativas en el área de matemática, para lo cual se trabajó con una población y muestra representativa constituida por 42 estudiantes del quinto grado de educación primaria de la Institución Educativa $\mathrm{N}^{\circ} 16139$, Sallique - Jaén, distribuidos en dos grupos: el grupo experimental con 22 alumnos (sección A) y el control con 20 alumnos (sección B). Se aplicó estrategias cognitivas y metacognitivas para mejorar capacidades significativas en el área de matemática y se logró elevar el nivel básico en $45.5 \%$ y 40,9\% en el nivel suficiente, permitiendo de esta manera desarrollar capacidades matemáticas en las dimensiones: número, relaciones y operaciones, geometría y medición, y estadística, así como en la asimilación e interiorización de los pasos para la comprensión, elaboración, aplicación y revisión del problema, reconociendo y utilizando los procesos cognitivos y metacognitivos en la resolución de problemas. Para probar la hipótesis se optó por el diseño cuasi-experimental de tipo pre test y post test, cuyo instrumento utilizado fue construido y validado por expertos. Para el análisis de datos se utilizó las pruebas estadísticas " $t$ " de Student y el SPSS 15. Se concluye que las estrategias aplicadas fueron eficientes y eficaces dado que los estudiantes lograron desarrollar capacidades matemáticas en las dimensiones: número, relaciones y operaciones; geometría y medición, y estadística.

Palabras clave: Estrategias, capacidades matemáticas, resolución de problemas.

\begin{abstract}
This investigation has such a purpose to apply cognitive and metacognitive strategies to develop significant capacities in the area of mathematics, we worked with a population and a representative sample constituted for 42 students of the fifth grade of primary education of the Educative Institution $N^{0} 16139$, Sallique - Jaén, distributed in two groups: the experimental group with 22 students (section "A") and the one of control with 20 students ("B"). We applied cognitive and metacognitive strategies to improve significant capacities in the area of mathematics and we achieved increase the basic level at $45.5 \%$ y $40,9 \%$ in the sufficient level, thus allowing to develop mathematics skills in the dimensions: number, relations and operations, geometry and measurement, and statistics, also in the on assimilation and internalization of the steps for understanding, processing, application and review of the problem, recognizing and using cognitive and metacognitive processes in problem solving. To test the hypothesis is chose for quasi-experimental design of pre-test and post-test type whose instrument used was constructed and validated by experts. For data analysis, we used the test statistics T of "Student" and the SPSS 15. We conclude that the strategies used were efficient and effective because students were able to develop mathematics skills in the dimensions: number, relations and operations, geometry and measurement, and statistics.
\end{abstract}

Keywords: Strategies, mathematics skills, problem solving.

\footnotetext{
Universidad César Vallejo. Lambayeque.

${ }^{2}$ Universidad Nacional de Jaén. Cajamarca.

${ }^{a}$ Licenciado en Educación, ${ }^{b}$ Licenciado en Matemática.
} 


\section{INTRODUCCIÓN}

Actualmente la enseñanza de la matemática en educación primaria se ve reducida a un trabajo mecánico, rutinario y descontextualizado, porque prevalece el memorizar procedimientos y fórmulas que los estudiantes no comprenden; de igual manera, se suelen resolver operaciones básicas con algoritmos que desnaturalizan el verdadero significado y razón de ser de las operaciones, motivo por la cual en el nivel primario muestran deficiencias en elárea de matemática.

Como plantea (Beltrán, 1995) las estrategias cognitivas son una especie de reglas o procedimientos intencionales que permiten al sujeto tomar las decisiones oportunas de cara a conformar las acciones que caracterizan el sistema cognitivo. Las dos tareas cognitivas más elementales conciernen a la adquisición y al procesamiento de la información.

Según el (Ministerio de Educación del Perú, 2006), la metacognición es el conjunto de procesos mentales que utilizamos cuando guiamos la manera como llevamos a cabo una tarea o actividad. Dicha actividad puede ser solucionar ejercicios y problemas matemáticos o investigar un tema matemático en una enciclopedia. La metacognición nos sirve para guiar nuestra ejecución con el fin de hacerla de manera más inteligente, comprendiendo bien lo que hacemos y controlando nuestras estrategias.

La capacidad de resolución de problemas implica que el estudiante manipule los objetos matemáticos, active su propia capacidad mental, ejercite su creatividad, reflexione y mejore su proceso de pensamiento, al aplicar y adaptar diversas estrategias matemáticas en diferentes contextos. La capacidad para plantear y resolver problemas, dado el carácter integrador de este proceso, posibilita la interacción con las demás áreas curriculares contribuyendo al desarrollo de otras capacidades; asimismo, posibilita la conexión de las ideas matemáticas con los intereses y experiencias del estudiante. Para fines curriculares, el área de matemática se organiza en función de: números, relaciones y operaciones, geometría y medición, y estadística (Ministerio de Educación, 2009).

Según (Polya, 1945) la resolución de problemas matemáticos consta de cuatro fases: comprensión del problema, concepción de un plan, ejecución del plan y visión retrospectiva. Considerándose fundamentales para cimentar algunos puntos del presente estudio.

Al respecto (Miguel de Guzmán, 1991) dice que las cuatro fases de Polya, orienta y anima al resolutor en los siguientes aspecto: familiarizarse con el problema en la cual el estudiante o el resolutor trata de entender a fondo la situación, en seguida pasa a la búsqueda de estrategias, que le permitan resolver el problema, desde diferentes puntos de vista, seguidamente el resolutor debe llevar a cabo la estrategia plancada, evaluándola a través de preguntas que evalúen el proceso seguido, y finalmente se llega al proceso de revisión y de establecer conclusiones, exanninando todo el camino, preguntándose ¿Cómo se ha llegado a la solución? O bien, ¿Por qué no se llegó?, tratar de entender no sólo que el procedimiento funciona, sino por qué funciona, mirar si se encuentra un camino más simple y reflexionar sobre su propio proceso de pensamiento (proceso metacognitivo) y sacar consecuencias para el futuro.

El mismo (Piaget, 1963) definió a la operación mental como acción interiorizada que modifica el objeto de conocimiento. El poder identificar las operaciones mentales o procesos cognitivos que ocurren en nuestra mente cuando hacemos uso de una capacidad, es de mucha utilidad para mejorar los aprendizajes, así como, para hacer más eficiente y elevar la calidad del trabajo o actividad que nos disponemos a realizar. Para Piaget el estudiante construye activamente sus conocimientos, en el sentido de que no los acumula, y más bien los transforma, los configura y les da significado acorde en el objeto de su aprendizaje. Dicha construcción la lleva a cabo, fundamentalmente, mediante dos procesos: el proceso de asimilación y el de acomodación.

En este contexto, surge la siguiente interrogante ¿Cómo desarrollar capacidades significativas en el área de matemática en los niños y niñas del quinto grado de educación primaria en la Institución Educativa $N^{\circ} 16139$, Sallique - Jaén? y como propósito aplicar estrategias cognitivas y metacognitivas para desarrollar capacidades significativas en el área de matemática en los niños y niñas del quinto grado de educación primaria en la Institución Educativa $\mathrm{N}^{\circ}$ 16139, Sallique-Jaén.

\section{MATERIALYMÉTODOS}

\section{Hipótesis \\ Hipótesis alterna}

$\mathbf{H}_{\mathbf{a}}$ : La aplicación de estrategias cognitivas y metacognitivas desarrollan significativamente capacidades en el área de matemática en los niños y niñas del quinto grado de educación primaria en la Institución Educativa $\mathrm{N}^{\circ}$ 16139, Sallique - Jaén.

\section{Hipótesis nula}

$\mathbf{H}_{\mathbf{0}}$ : La aplicación de estrategias cognitivas y metacognitivas no desarrollansignificativamente 
capacidades en el área de matemática en los niños y niñas del quinto grado de educación primaria en la Institución Educativa $\mathrm{N}^{\circ} 16139$, Sallique-Jaén.

\section{EI Tipo de estudio realizado.}

Es aplicativa, descriptiva y explicativa.

\section{Diseño de estudio}

Este estudio es cuasi-experimental y consistió en seleccionar dos grupos: uno experimental y otro de control, en ambos casos se aplicó una primera prueba o pre test a cada grupo. Seguidamente al grupo experimental se aplicó las estrategias cognitivas y metacognitivas, al grupo de control las actividades de aprendizajes normales; $y$ al culminar con la ejecución de las estrategias de intervención a los dos grupos se les aplicó una segunda prueba o post test para contrastar, discutir y analizar los resultados obtenidos.

\section{Población y muestra}

La población y muestra estuvo constituida por todos los estudiantes del quinto grado de educación primaria de la Institución Educativa $\mathrm{N}^{\circ} 16139$, Sallique - Jaén - 2011, equivalente a 42 niños y niñas (100\% de estudiantil del quinto año). Los grupos fueron agrupados: grupo experimental "A" (22 estudiantes) y grupo control "B" (20 estudiantes).

Tabla 1. Técnicas e instrumentos de recolección de datos

\begin{tabular}{ccl}
\hline Variables & Técnicas & \multicolumn{1}{c}{ Instrumentos } \\
\hline $\begin{array}{c}\text { Dependiente } \\
\text { Capacidades en el } \\
\text { área de matemática. }\end{array}$ & Prueba o test & $\begin{array}{l}\text { Test de evaluación (pre test), dirigida a los estudiantes del } \\
\text { quinto grado de educación primaria, con la finalidad de } \\
\text { obtener información acerca del nivel de desarrollo de } \\
\text { capacidades en el área de matemática. }\end{array}$ \\
$\begin{array}{c}\text { Independiente } \\
\text { Estrategias cognitivas } \\
\text { y melacognitivas. }\end{array}$ & Observación & $\begin{array}{l}\text { Lista de cotejo, para valorar las estrategias cognitivas y } \\
\text { metacognitivas. }\end{array}$ \\
& Entrevista & $\begin{array}{l}\text { Guía de entrevista de profundidad, para verificar la } \\
\text { influencia de las estrategias cognitivas y metacognitivas en } \\
\text { el desarrollo capacidades en el área de matemática. }\end{array}$ \\
\hline
\end{tabular}

Asimismo, se utilizó técnicas de gabinete para el análisis y evaluación de documentos de gestión pedagógica, administrativa e institucional: Proyecto Educativo Institucional, Proyecto Curricular de Centro, Programación Curricular Anual, Unidades didácticas (unidades, proyecto y módulos de aprendizajes, nóminas de matrículas, registros auxiliares y oficiales), diarios de clase de los docentes del área de matemática y se recogió información a través de Fichas bibliográficas: textuales y de resumen.

\section{Métodos de análisis de datos}

Los datos obtenidos a través de los instrumentos aplicados, se organizaron en tablas de distribución de frecuencias: absolutas y porcentuales, a partir de las cuales se elaboraran las gráficas respectivas. Para establecer las diferencias entre los aprendizajes promedio de los alumnos en las habilidades consideradas calculados al final de la investigación (post test), se aplicó la prueba estadística " $\mathrm{t}$ " de Student con un nivel de significación del 5\% $\quad(\alpha=$ $0,05)$.

\section{RESULTADOS}

Tabla 2. Resumen de los consolidados totales de las tres dimensiones (número, relaciones y operaciones; geometría y medición; y estadística).

\begin{tabular}{|c|c|c|c|c|c|c|c|c|}
\hline \multirow{4}{*}{$\begin{array}{c}\text { ESCALA DE } \\
\text { VALORACIÓN }\end{array}$} & \multicolumn{8}{|c|}{ Grupos } \\
\hline & \multicolumn{4}{|c|}{ Experimental - Aula "A" } & \multicolumn{4}{|c|}{ Control- Aula "B" } \\
\hline & \multicolumn{2}{|c|}{ Pre Test } & \multicolumn{2}{|c|}{ Post Test } & \multicolumn{2}{|c|}{ Pre Test } & \multicolumn{2}{|c|}{ Post Test } \\
\hline & Alum & $\%$ & Alum & $\%$ & Alum & $\%$ & Alum & $\%$ \\
\hline Bajo previo & 16 & 72,8 & 00 & 00,0 & 15 & 75,0 & 8 & 40,0 \\
\hline Previo & $\mathbf{0 3}$ & 13,6 & 00 & 00,0 & 05 & 25,0 & 7 & 35,0 \\
\hline Básico & $\mathbf{0 3}$ & 13,6 & 13 & 59,1 & 00 & 00,0 & 5 & 25,0 \\
\hline Suficiente & 00 & 00,0 & 09 & 40,9 & 00 & 00,0 & $\mathbf{0}$ & 00,0 \\
\hline Total & 22 & 100 & 22 & 100 & 20 & 100 & 20 & 100 \\
\hline
\end{tabular}




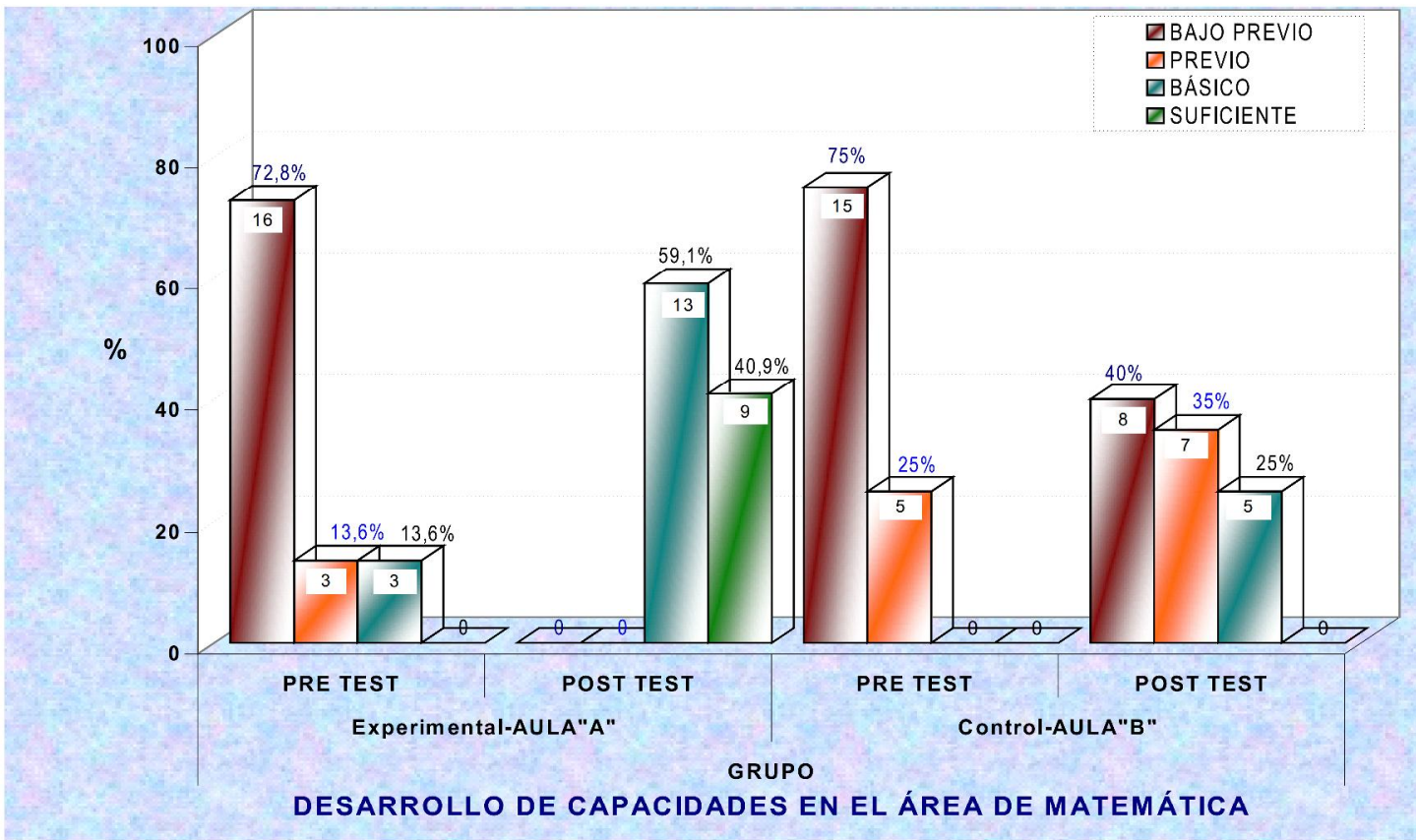

Figura 1. Resumen de los consolidados totales de las tres dimensiones (número, relaciones y operaciones; geometría y medición; y estadística). Fuente: Test aplicado por los investigadores.

Como se aprecia en la Tabla 2 y Figura 1 el desarrollo de capacidades en el área de matemática en las dimensiones, Número, Relaciones y Operaciones; Geometría y Medición; y Estadística, en los estudiantes del quinto grado de educación primaria de la I.E No 16139 , Sallique -Jaén, (Sección $\Lambda$ grupo experimental); de acuerdo a los resultados del pre test se ubican en el nivel por debajo del previo con un 72,8 \%; en el nivel previo $13,6 \%$, nivel básico $13,6 \%$, y ningún estudiante ha logrado el nivel suficiente; pero, después de haber aplicado las estrategias cognitivas y metacognitivas, los resultados del post test son los siguientes: 59,1 \% nivel Básico, 40,9\%, nivel suficiente, y ningún alumno se encuentra en el nivel por debajo del previo y previo. En lo referente al grupo de control (Sección B), los resultados relacionados al pre test se expresan de la siguiente manera: $75 \%$ de estudiantes se ubican en el nivel por debajo del previo, sólo el $25 \%$ en el nivel previo; de igual forma, los resultados del post test, se observa que el $40 \%$ de estudiantes siguen presentando un nivel por debajo del previo, un $35 \%$ se encuentran en el nivel previo, y $25 \%$ en el nivel básico; por tanto, ningún estudiante alcanza el nivel suficiente.

\section{DISCUSIÓN}

El análisis y discusión de los resultados tanto del pre test como del post test del grupo experimental (Aula "A" - Tabla 2) podemos observar que después de haber aplicado las estrategias cognitivas y metacognitivas se evidencia un avance significativo en el post test. Esto significa que los estudiantes lograron desarrollar capacidades matemáticas para aplicar propiedades en la resolución de operaciones combinadas; establecer relaciones entre los números y las operaciones para resolver problemas, identificar y encontrar regularidades; resolver y formular problemas que implican adición y sustracción de fracciones heterogéneas; identificar e interpretar prismas, reconocer figuras geométricas; representar, calcular y argumentar perímetros y áreas; organizar, interpretar y argumentar datos presentados en gráficos de barras, poligonales y circulares a través del uso de un lenguaje matemático adecuado. Asimismo, se asimilaron los procesos de resolución de problemas propuestos por Polya y De Guzmán, reconociendo los procesos cognitivos y metacognitivas que aplicaron y asumieron un rol participativo y autónomo en la construcción de sus aprendizajes. En contraste, en el grupo de control (Sección "B" - Tabla 2); ningún estudiante alcanza el nivel suficiente.

La intención de desarrollar capacidades matemáticas en los estudiantes se fundamenta en los aportes de (Santaló, 1994, citado por Pérez de Zapata, 1997), y (Rico, 2000), quienes consideran que el proceso formativo de la matemática se expresa en el desarrollo de capacidades, entre otras 
el razonamiento lógico, simbolización, abstracción, rigor y precisión que caracterizan al pensamiento formal; de igual forma, está relacionada con el desarrollo de habilidades cognoscitivas abstractas, tales como la capacidad para razonar, abstraer, deducir, entre otras, y permite desarrollar la capacidad de pensar, influyendo en la formación de la inteligencia. Por otro lado, (Piaget, 1963) define a la operación mental como "acción interiorizada que modifica el objeto de conocimiento"; en ese sentido cree que el poder identificar las operaciones mentales o procesos cognitivos que ocurren en nuestra mente cuando hacemos uso de una capacidad, es de mucha utilidad para mejorar los aprendizajes, así como, para hacer más eficiente y elevar la calidad del trabajo o actividad que nos disponemos a realizar.

En base a los resultados anteriores, hallamos relación con la investigación de (Salas, 2008) titulada Adaptación y aplicación del programa de desarrollo de estrategias metacognitivas Aprendo a pensar"en el aprendizaje de la aritmética en alumnas del $1^{\circ}$ grado de educación secundaria, realizada en la Universidad Nacional Mayor de San Marcos, que establece que las estrategias metacognitivas sirven para que los estudiantes aprendan a planificar, controlar y valorar sus procesos de pensamiento con el fin de mejorar su rendimiento en el área de matemática y, asimismo, aprendan a conocer sus propios mecanismos de aprendizaje y a rentabilizar mejor sus esfuerzos.

Debido al uso de ciertos materiales didácticos que se utilizó como medios para trabajar esta dimensión asumimos el trabajo de investigación de (Díaz, 2007), titulada "Estrategias y materiales educativos para desarrollar los procesos del pensamiento matemático en los alumnos del cuarto grado de educación secundaria de la I.E. César Vallejo-Cumba, realizada por el Instituto de Educación Superior Pedagógico Público "Víctor Andrés Belaunde", Provincia de Jaén, Región Cajamarca, que concluyen que la construcción del conocimiento es un proceso de representación mental de la información a través de imágenes y conceptos, manipulaciones mentales de la información por medio de operaciones o destrezas intelectuales, disposiciones y actitudes hacia la información, que facilitan o dificultan su representación y manipulación mental.
De la misma forma señalan que el uso de recursos pedagógicos innovadores como juegos educativos y materiales educativos en las clases de matemática, ayuda y facilitan a los alumnos, a que puedan abstraer y definir propiedades y conceptos matemáticos. Por lo tanto, la aplicación de diversas estrategias cognitivas, metacognitivas y materiales educativos permitieron el desarrollo de los procesos del pensamiento matemático.

Las afirmaciones que anteceden se correlacionan con lo propuesto por (Pifarre y Sanuy, 2001), en su tesis titulada "La enseñanza de estrategias de resolución de problemas matemáticos en la Educación Secundaria Obligatoria (ESO)": un ejemplo concreto, en los estudiantes de tercer grado de la Enseñanza Secundaria Obligatoria en la ciudad de Lleida (España), consideran que en toda propuesta didáctica debe incorporarse cuatro elementos fundamentales: a) contextualizar los problemas a resolver por el alumno en situaciones cotidianas de su entorno; b) utilizar métodos de enseñanza que hagan visibles las acciones para resolver un problema, proceso poco conocido desde el punto de vista del alumno; c) diseñar diferentes tipos de materiales didácticos que guíen la selección, la organización, la gestión y el control de los diferentes procedimientos para resolver un problema; y d) crear espacios de discusión y de reflexión alrededor de este proceso, como por ejemplo, el trabajo en pequeños grupos o en parejas. Por su parte, las estrategias cognitivas y metacognitivas fueron contextualizadas y respondieron pertinentemente con la realidad sociocultural y académica de los estudiantes, además, tuvieron un carácter significativo y funcional, permitiendo que los alumnos sean capaces de aplicar propiedades para desarrollar operaciones combinadas; configurar situaciones problemáticas en un diagrama adecuado y poder organizar contenidos; encontrar de manera gráfica (geométrica) regularidades en diferentes situaciones numéricas; $y$, desarrollar la capacidad de interpretar la construcción de un gráfico de barras y de ella inferir y argumentar información relevante de ésta.

\section{CONCLUSIONES}

Con la aplicación de estrategias cognitivas y metacognitivas se logró mejorar significativamente 
capacidades en el área de matemática en los niños y niñas del quinto grado de educación primaria en la Institución Educativa $\mathrm{N}^{\circ}$ 16139, Sallique - Jaén, elevándose del 13,6\% al 59,1\% el nivel básico y de $0 \%$ al $40,9 \%$ el nivel suficiente, permitiendo desarrollar capacidades matemáticas en las dimensiones: número, relaciones y operaciones, geometría y medición, y estadística. De esta manera se mejoró en la asimilación e interiorización de los pasos para la comprensión, elaboración, aplicación y revisión del problema, reconociendo y utilizando los procesos cognitivos y metacognitivos en la resolución de problemas.

\section{AGRADECIMIENTO}

A la Comisión Organizadora de la Universidad Nacional de Jaén por confiar en sus docentes e investigadores para publicar sus trabajos de investigación en la Revista Pakamuros y a nuestra familia por su apoyo constante y sincero.

\section{REFERENCIAS BIBLIOGRÁFICAS}

Beltrán, J. 1995. Conocimiento, pensamiento e interacción social. Madrid: Síntesis, S.A.

Beltrán, J. 1998. Procesos, estrategias y técnicas de aprendizaje. Madrid: Síntesis, S.A.

De Guzmán, M.1991. Enseñanza de las Ciencias y la Matemática. España.

Díaz, E. 2007. Estrategias y materiales educativos para desarrollar los procesos del pensamiento matemático en los alumnos del cuarto grado de educación secundaria de la I.E. "César Vallejo"Cumba, en IFSPPVAB. Jaén, Perú.

Ministerio De Educación. 2006. Guía para el desarrollo de los procesos metacognitivos. Kinko's Impresores S.A.C. Primera Edición. Lima-Perú.

Ministcrio De Educación. 2006. Guía para cl desarrollo de la capacidad de solución de problemas. Fimart S.A.C. Primera Edición. Lima.

Ministerio De Educación. 2009. Diseño Curricular Nacional de la Educación Básica Regular de Primaria de Menores. Área matemática. Dirección nacional de educación inicial y primaria.

Piaget, J. 1963. Theorigins of intelligence in children. New York.
Pifarré, M y J. Sanuy. 2001. La enseñanza de estrategias de resolución de problemas matemáticos en la ESO (Educación Secundaria Obligatoria): un ejemplo concreto, en los estudiantes de tercer grado de la Enseñanza Secundaria Obligatoria en la ciudad de Lleida. España.

Polya, G. 1945. Cómo plantear y resolver problemas. Editorial Trillas. México.

Rico, L. 2000. Consideraciones sobre el currículo escolar de matemáticas. Universidad los Andes. Disponible en: http:ued.uniandes.edu.co/ servidor/ued/revistaema/voll num1/ailrico .html.Colombia (Consultado el 24 de julio, 2011).

Salas, R. 2008. Adaptación y aplicación del programa de desarrollo de estrategias metacognitivas "Aprendo a pensar" en el aprendizaje de la aritmética en alumnas del $1^{\circ}$ grado de educación secundaria. Facultad de psicología. Universidad Nacional Mayor de San Marcos, Lima -Perú.

Santaló, L. 1994 (citado por Pérez de Zapata, 1997). Seminario internacional de medición de la calidad en educación (Sistemas de evaluación y medición de la calidad de la educación). Ministerio de Educación. Piura-Lima-Cusco.

\section{Correspondencia}

Carlos Alberto Núñez Rivas. San Leandro $N^{0}$ - Jaén-Cajamarca. canuri_30@hotmail.com 